\title{
Expression of PAX5 in CD20-positive multiple myeloma assessed by immunohistochemistry and oligonucleotide microarray
}

\author{
Pei Lin ${ }^{1}$, Mustafa Mahdavy ${ }^{1}$, Fenghuang Zhan ${ }^{2}$, Hua-Zhong Zhang ${ }^{3}$, Ruth L Katz ${ }^{3}$ and \\ John D Shaughnessy ${ }^{2}$ \\ ${ }^{1}$ Department of Pathology, University of Arkansas for Medical Science, AR, USA; ${ }^{2}$ Lambert Laboratory of \\ Myeloma Genetics, Little Rock, AR, USA and ${ }^{3}$ Department of Pathology, The University of Texas-MD \\ Anderson Cancer Center, Houston, TX, USA
}

\begin{abstract}
Silencing of PAX5 gene by upregulation of B-lymphocyte-induced maturation protein-1 (PRDM1) is essential for terminal differentiation of $B$ cells to plasma cells. To investigate PAX5 gene expression and its protein product, B-cell-specific activator protein (BSAP), in a subgroup of multiple myeloma characterized by CD20 expression, we studied PAX5/BSAP by immunohistochemistry in 25 cases of myeloma, all expressing moderate to strong CD20 by flow cytometric analysis, and correlated the results with PAX5 and PRDM1 mRNA levels analyzed by the Affymetrix HuGeneFL GeneChip microarray in 17 cases. Using paraffin-embedded bone marrow biopsy sections, we found PAX5/BSAP was expressed in $72 \%$ (18/25) of cases overall with an intensity ranging from weak $(10,56 \%)$ to strong $(8,44 \%)$. PAX5/BSAP was negative in 10 randomly selected CD20-negative myelomas included as negative controls. PAX5 mRNA levels correlated inversely with that of PRDM1 in both CD20-positive and CD20-negative myelomas and failed to predict the expression levels of PAX5/BSAP, suggesting that detected PAX5/BSAP likely represents remnant of earlier stage of development. We conclude that CD20-positive myelomas expressing PAX5/BSAP can present as a diagnostic pitfall mimicking B-cell neoplasms with plasmacytoid differentiation.

Modern Pathology (2004) 17, 1217-1222, advance online publication, 11 June 2004; doi:10.1038/modpathol.3800169
\end{abstract}

Keywords: PAX5; PRDM1; CD20; multiple myeloma; affymetrix microarray

PAX5 is a member of the paired domain family of transcription factors that encodes for B-cell-specific activator protein (BSAP). PAX5/BSAP plays a pivotal role in B-cell lineage commitment and differentiation by targeting several B-cell-related genes including CD19 and CD79a (mb-1). ${ }^{1-3}$ B cells from pro-B to mature $\mathrm{B}$ stage express PAX5. Silencing of PAX5 at the plasma cell stage is considered to be critical for maturation of B cells to plasma cells and is mediated through upregulation of B-lymphocyte-induced maturation protein-1 (PRDM1), a transcriptional repressor encoded by the gene PRDM1. ${ }^{2,4}$

Previous studies of large series of T- and B-cell lymphomas and leukemias by immunohistochemis-

Correspondence. Current address: Dr Pei Lin, MD, Department of Hematopathology, Box 72, University of Texas-MD Anderson Cancer Center, Houston, TX, 77030, USA.

E-mail: peilin@mdanderson.org

Received 3 November 2003; revised and accepted 25 March 2004; published online 11 June 2004 try have shown that PAX5/BSAP is consistently expressed by precursor and mature B-cell lymphomas or leukemias but not by multiple myeloma or solitary plasmacytomas. ${ }^{5}$ Furthermore, the expression of PAX5/BSAP correlates strongly with CD20 expression. Thus, PAX5/BSAP has been considered an excellent marker for distinguishing B-cell neoplasms, particularly chronic lymphocytic leukemia with plasmacytoid features or lymphoplasmacytic lymphoma/Waldenstrom macroglobulinemia, from plasma cell neoplasms. About 10-15\% of myelomas express CD20. ${ }^{6}$ The expression patterns of PAX5 and PRDM1 in CD20-positive myeloma are unknown.

We studied PAX5/BSAP expression in CD20positive myelomas by immunohistochemistry using bone marrow biopsy samples. We also correlated the results with the mRNA levels of $P A X 5$ and PRDM1 analyzed by the Affymatrix HuGeneFL microarray using CD138 purified plasma cells from the corresponding bone marrow aspirates. 


\section{Materials and methods}

The files of Department of Pathology were searched for cases of multiple myeloma that expressed CD20 between January 2001 and March 2002. CD20 expression was determined by flow cytometry immunophenotypic analysis of bone marrow aspirate material. The diagnosis of myeloma was confirmed by reviewing medical records and diagnostic bone marrow biopsy samples. Each patient was staged according to the Salmon and Durie system ${ }^{7}$ and each tumor was graded according to the Bartl system. ${ }^{8}$ Conventional cytogenetic analysis was performed on the bone marrow aspirate material using techniques described before. ${ }^{9}$ We excluded cases with a monoclonal IgM on serum protein electrophoresis and immunofixation electrophoresis.

Flow cytometric immunophenotyping was performed on whole bone marrow aspirates anticoagulated with EDTA using methods described before. ${ }^{10}$ Briefly, after red cell lysis with ammonium chloride, the cells were resuspended and fixed with $1 \%$ methanol-formaldehyde and incubated with a panel of monoclonal antibodies specific for CD138PE (Immunotech, Marseille Cedex, France), CD138FITC, CD138-PC5, CD45-FITC, CD117-PE, CD20FITC, CD56-PE, and CD19-PE (Becton Dickinson Immunocytometry Systems, San Jose, CA, USA). The clonality of plasma cells was determined by dual analysis of immunoglobulin $\kappa$ and $\lambda$ light chains (Dako, USA) and DNA ploidy. Only rare contaminating normal plasma cells $(<1 \%)$ were found. All analyses were performed on a FACScan (Becton Dickinson, Mountain View, CA, USA) using Cell-quest software (Becton Dickinson).

Myeloma cells were first identified by their light scattered distribution and reactivity pattern to CD138, CD38 and CD45. CD20 expression was then determined by analyzing the gated region using CD138 and CD20. The intensity of CD20 expression was graded as weak, moderate or strong. For purpose of this study, we only included patients with moderate to bright expression of CD20, defined as a positive population at least 1 log brighter than the negative control.

PAX5 and PRDM1 mRNA levels were analyzed by the HuGeneFL GeneChip microarray (Affymetrix, Santa Clara, CA) using CD138 selected plasma cells from corresponding bone marrow aspirates. RNA extraction was followed by synthesis of cDNA and biotinylated cRNA and then hybridized to the oligonucleotide array. The expression levels were determined by fluorescence intensity and analyzed by Affymetrix 3.3 software as described before. ${ }^{11}$

Sections of formalin-fixed paraffin-embedded bone marrow biopsy were stained with monoclonal antibody specific for PAX5/BSAP (1:35, Transduction Laboratories, BD Biosciences, San Jose, CA, USA). Areas with large clusters or sheets of myeloma cells were assessed for staining intensity.
Immunostain with monoclonal antibody specific for CD138 (1:20, Dako, Carpenteria, CA, USA) was also performed in a subset of cases to assess the extent of plasma cell infiltrate. In all, 10 cases of randomly selected CD20-negative myelomas were included as negative controls.

The staining intensity of PAX5/BSAP was measured by computerized quantitative image analysis using a SAMBA 4000 Cell Image Analysis System (SAMBA Technologies, Meylan, France). The image analyzer is a PC-based integrated system for densitometric, morphometric, and colorimetric analysis of cells and tissues. It consists of a Nikon microscope with $\times 10, \times 20, \times 40$ objectives and a Sony $960 \mathrm{MD}$ 3-chip CCD camera, interfaced with a Dell computer (Dell Computer Corporation, Austin, TX, USA) equipped with a Matrox Meteor digitizer board (Matrox Electronic Systems Ltd, Dorval, and Quebec, Canada). Light and camera settings were standardized resulting in average background values of $210 \pm 5$ (mean \pm s.d., scale 0-255 from black to white) for the red, green and blue channels. Images were captured using $\times 20$ objective. Background subtraction was automatically performed on every tissue after storing an empty field of the slide. Analysis was performed after transformation of the red-green-blue (RGB) information to hue-saturation-intensity (HSI) information. The SAMBA software (Immuno-Analysis Version 4.28) enables the operator, after evaluating several fields on control slides, to set an intensity threshold value for the best discrimination between tissue and background. Separation between the hematoxylin counterstain (blue) and the immunostaining with DAB (brown) was achieved by setting a proper threshold on the hue value. The threshold for DAB-positive hues was obtained by evaluating several fields on positive and negative control slides for optimal separation between blue- and brown-stained areas.

Parameters measured were the mean optical density (MOD: the mean of optical densities measured over the labeled areas within the structure, proportional to the mean stain concentration), staining index (SI: the proportion of stained area relative to the total area of the structures) and the quick score (QS: $\mathrm{MOD} \times \mathrm{SI} / 100$ ). These parameters were generated from averaging the quantitative computerized image analysis data obtained from at least four fields of tumor components in each section. Based on the quick score, the staining intensity was graded as strong $(>10)$, weak $(<10$ and $>1.5)$, or negative $(<1.5)$.

\section{Results}

From 306 myeloma patients who were studied by flow cytometric immunophenotyping, we identified $28(9 \%)$ patients with moderate-strong CD20 expression on myeloma cells. The bone marrow biopsy specimens of 25 patients were available for immu- 

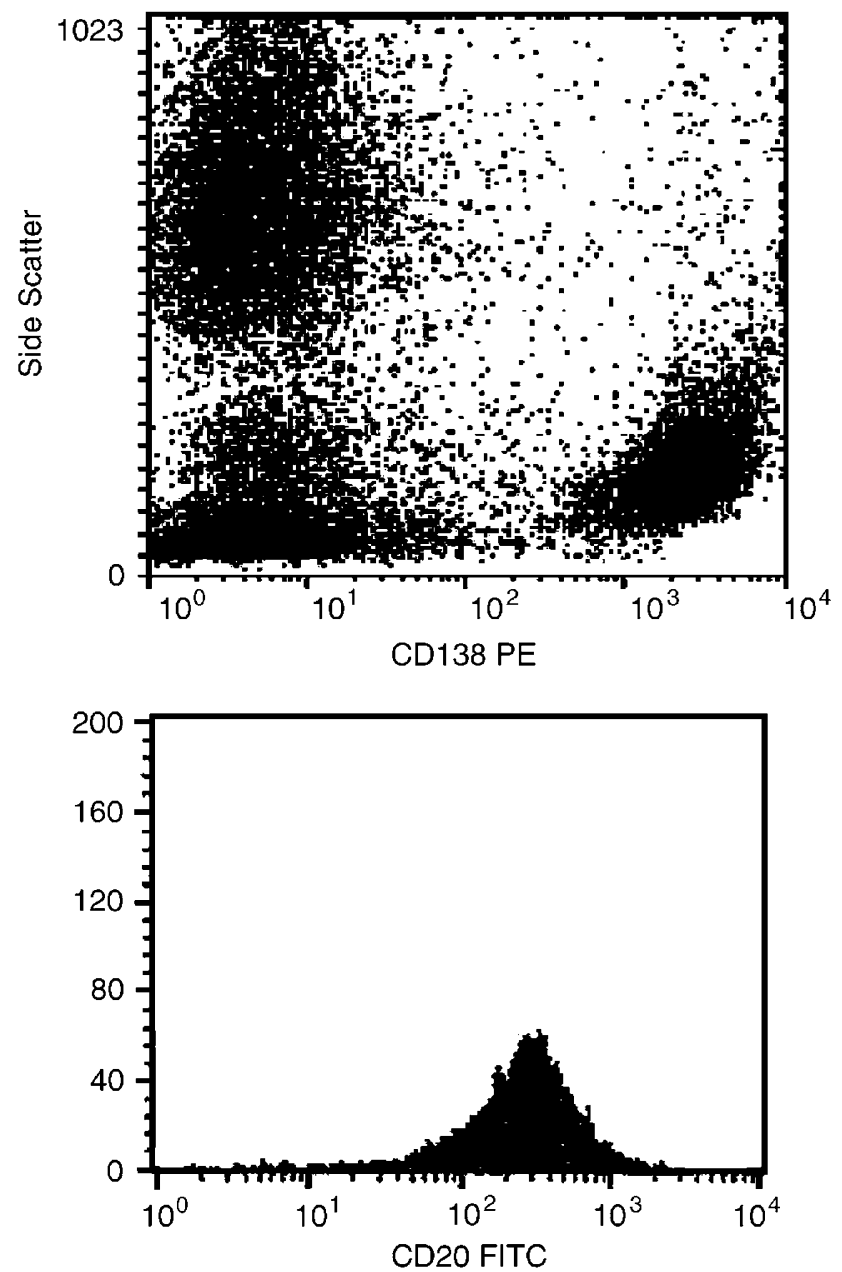

Figure 1 CD138 gating identifies a population of CD138 bright PCs that coexpress CD20 at moderate-strong intensity.

nostaining. CD20 was expressed by the entire plasma cell population in 23 patients and by $50 \%$ of plasma cells in two patients. The intensity of CD20 was strong in 21 cases and moderate in four cases (Figure 1). All cases were CD19 negative by flow cytometric immunophenotyping. The clinicopathological features of the 25 patients are summarized in Table 1 (Figure 2a).

\section{PAX5/BSAP Protein Expression}

PAX5/BSAP expression was detected in 18 (72\%) cases overall. The staining intensity was strong in eight $(44 \%)$ and weak in $10(56 \%)$ cases, respectively (Figure 2b). The quick score for cases with strong reactivity ranged from 10.32 to 19.06 (median 13.13). The quick score for cases with weak reactivity ranged from 1.50 to 3.59 (median 2.35). PAX5/BSAP was negative in seven cases of CD20positive myeloma and in all 10 cases of CD20negative myeloma.
Table 1 Clinicopathological features of 25 patients with CD20positive myeloma

\begin{tabular}{lc}
\hline Age (median) & $43-76$ year (58) \\
Gender & 12 male, 13 female \\
Salmon and Durie stage & 20 \\
Stage 3 & 3 \\
Stage 2 & 2 \\
Stage 1 & \\
Bartl Grade & 9 \\
1 & 15 \\
2 & 1 \\
3 & \\
Extent of bone marrow involvement (biopsy) & 8 \\
$20-50 \%$ & 17 \\
>50\% & \\
Type of monoclonal protein & 16 \\
IgG & 6 \\
IgA & 3 \\
Light chain only & \\
Karyotype & 13 \\
Diploid & 2 \\
Complex with $\mathrm{t}(11 ; 14)(\mathrm{q} 13 ; \mathrm{q} 32)$ & 1 \\
Complex with $\mathrm{t}(6 ; 14)(\mathrm{p} 21 ; \mathrm{q} 32)$ & 9 \\
Complex (others) & \\
\hline
\end{tabular}

Of the 17 cases that were studied by both immunohistochemistry and the Affymetrix GeneChip microarray, PAX5/BSAP was expressed strongly in four and weakly in eight cases.

\section{PAX5 and PRDM1 mRNA Expression}

$P A X 5$ and PRDM1 mRNA levels were available in 17 patients. The mean florescence intensity of PAX5 mRNA ranged from 60 to 817 (median 210). It was $<100$ in two patients, $100-500$ in 13 patients and $>500$ in two patients, respectively. In contrast, the mean florescence intensity of PRDM1 mRNA ranged from 1734 to 6216 (median 3298).

In the 10 patients with CD20-negative myeloma, the mean florescent intensity of PAX5 mRNA ranged from 99 to 565 (median 206). The corresponding PRDM1 levels were in the range of 1047-5167 (median 3078).

A strong inverse correlation between levels of PAX5 and PRDM1 mRNA expression was observed in both CD20-positive and CD20-negative myelomas (Figure 3). In addition, PAX5 mRNA did not predict the expression intensity of PAX5/BSAP as assessed by immunohistochemistry. PAX5 mRNA levels were low in both PAX5/BSAP-positive and -negative cases (Figure 4).

\section{Discussion}

PAX5 is a transcription factor that belongs to the paired box gene family. The predominant form of PAX5 (PAX5a) encodes for a $50 \mathrm{kDa}$ protein, PAX5 or BSAP that regulates B-cell differentiation through activation or suppression of different target genes. ${ }^{1}$ The binding sites of BSAP have been detected in the 
a
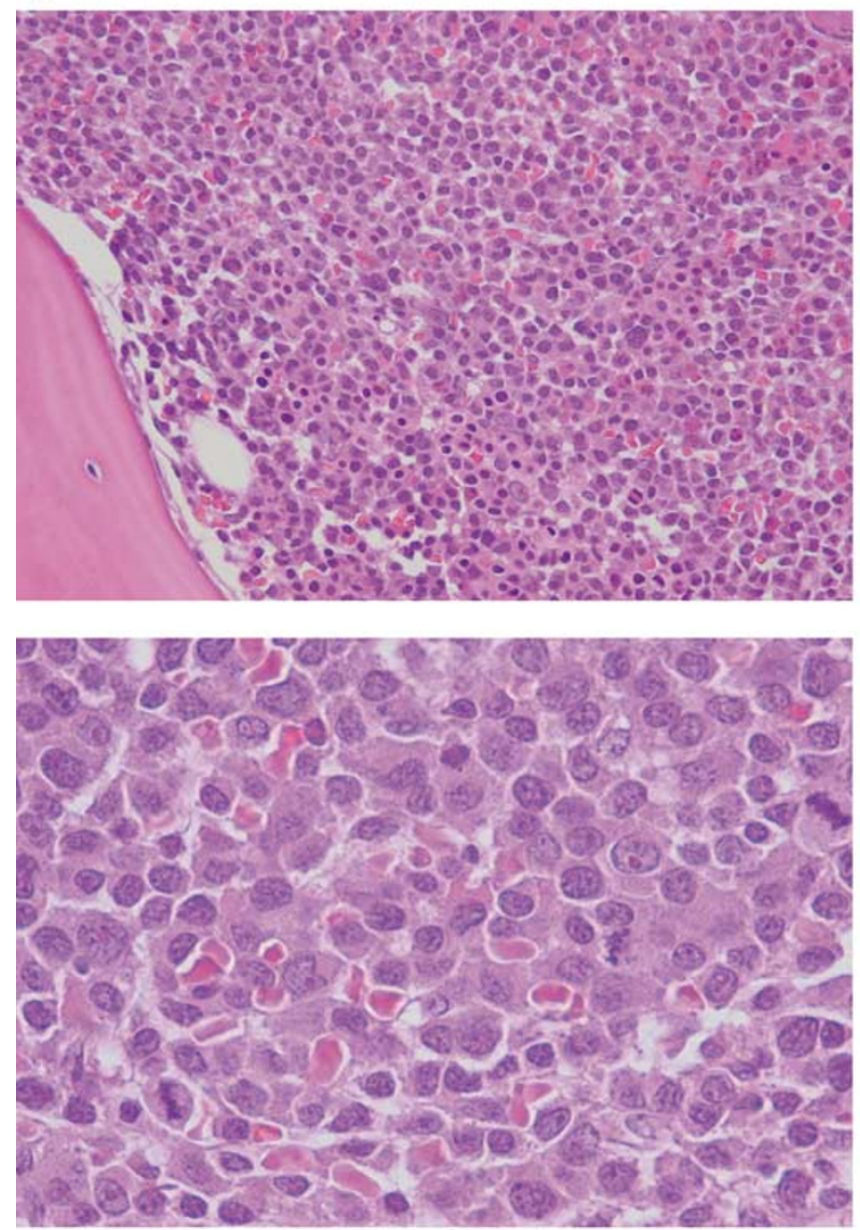

b
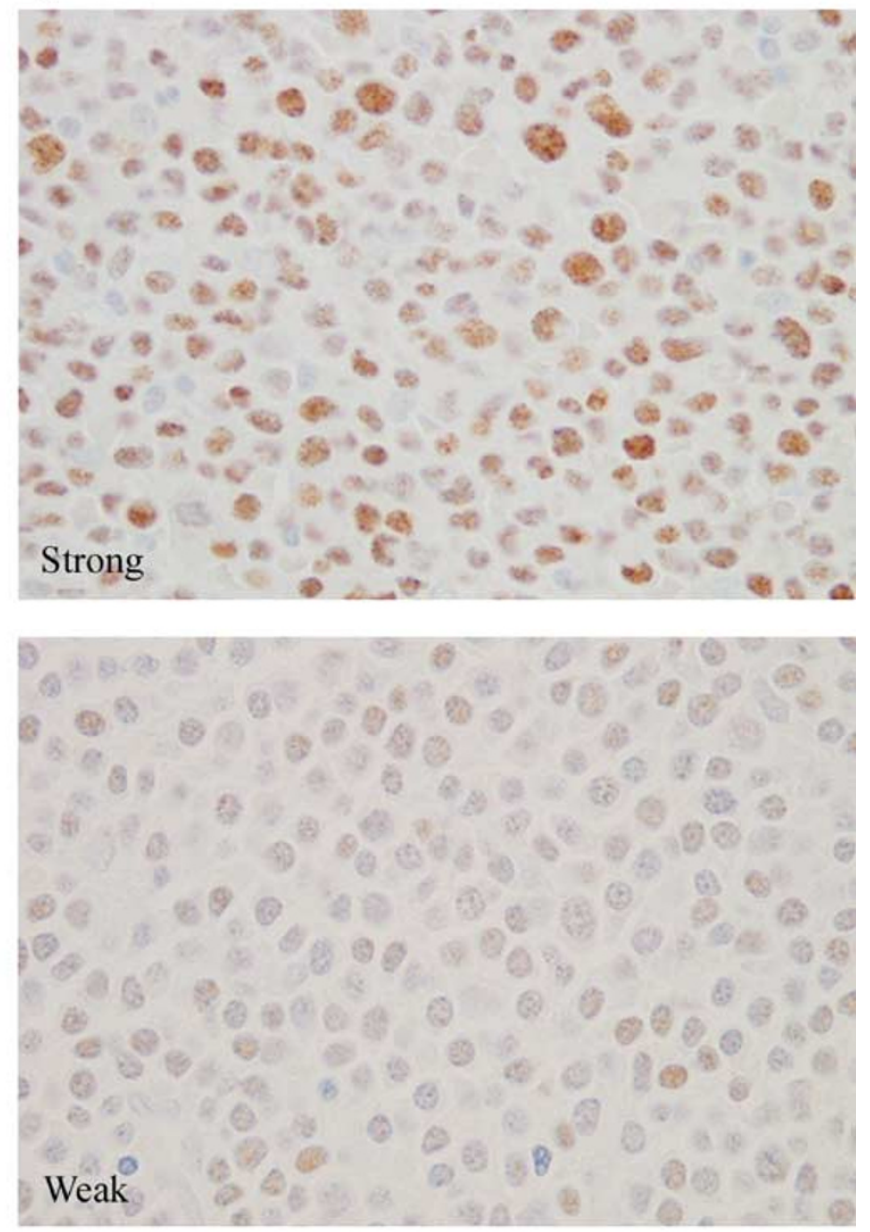

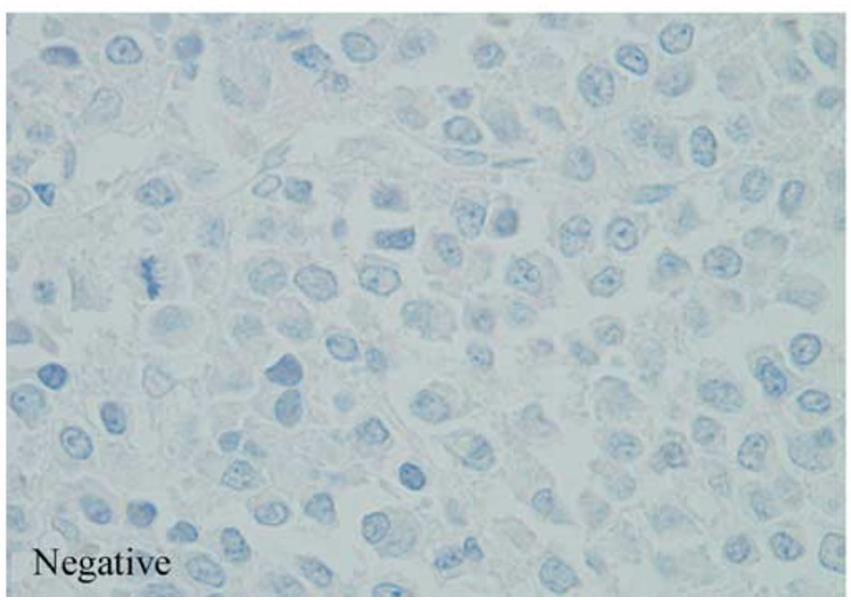

Figure 2 (a) A case of CD20-positive myeloma showing moderate cytologic atypia (Bartl grade 2) and increased mitosis. Hematoxylin and eosin, $\times 200$ (upper panel) and $\times 400$ (lower panel). This case was strongly and diffusely positive for PAX5/BSAP. (b) PAX5/BSAP immunostain performed on bone marrow biopsy was graded as strong, weak, or negative based on computerized quantitative imaging analysis $\times 400$.

promoter regions of CD19 and CD20. ${ }^{12,13}$ Studies of a mouse model indicate that PAX5 expression is required for commitment of hematopoietic progenitor cells to B-cell lineage and for differentiation of pro-B to mature B-cell stage until the point of terminal differentiation to plasma cells. Silencing of PAX5 at the plasma cell stage is generally considered prerequisite for differentiation of mature $\mathrm{B}$ cells to immunoglobulin-secreting plasma cells and is mediated through PRDM1, a transcriptional 
repressor encoded by a positive regulatory domain zinc-finger protein 1 gene (PRDM1). PRDM1 binds to the PAX5 promoter and represses $P A X 5$ transcription. ${ }^{2}$ It is expressed by all plasma cells and a subset of germinal center B cells (5-15\%) differentiating to plasma cells. ${ }^{14}$

In a large series of lymphomas and leukemias that included 39 cases of myeloma, ${ }^{5}$ Torlakovic et $a l^{5}$ found that PAX5/BASP was consistently negative in myeloma, and therefore serves as an excellent marker for distinguishing plasma cell neoplasms from B-cell neoplasms. Only two cases in their study had focal PAX5/BSAP positivity and both were also weakly positive for CD20. These two patients were found to have unusual clinical features characterized by absence of lytic bone lesions but presence of $>10 \%$ infiltrate in the bone marrow and $>3 \mathrm{~g} / \mathrm{l}$ serum monoclonal immunoglobulin. Other studies have described CD20-positive myeloma as frequently associated with $\mathrm{t}(11 ; 14)$, small mature morphology ${ }^{15}$ or plasma cell leukemia. ${ }^{16}$

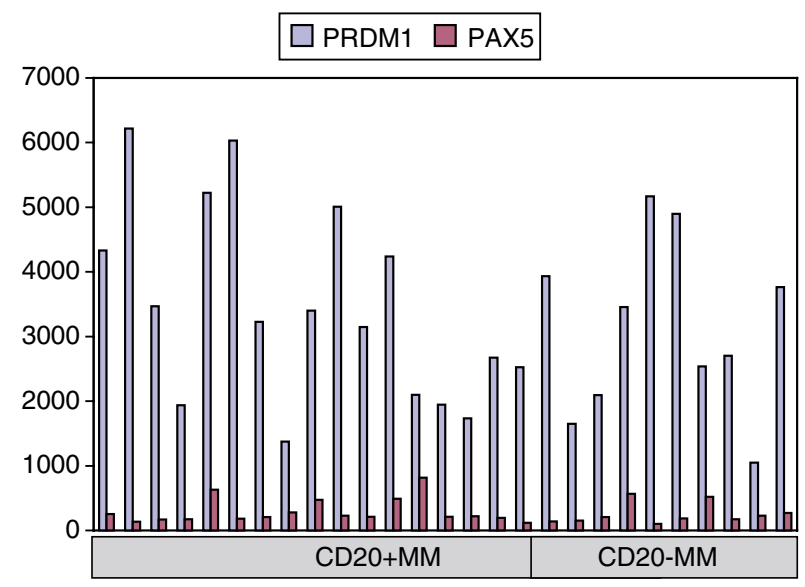

Figure 3 mRNA levels of PAX5 and PRDM1 in 17 cases of CD20 positive and 10 cases of CD20 negative myeloma analysed by HuGeneFL GeneChip microarray.
The myeloma cases in this study cohort presented with a range of cytologic atypia, type of M-protein, and karyotypic aberrations. Most patients had an advanced stage of disease, intermediate cytological grade and serum monoclonal IgG. Diploid karyotype was the most common finding. Abnormal karyotypes were characterized by complex changes with infrequent occurrence of $\mathrm{t}(11 ; 14)$.

We confirmed the findings reported by Torlakovic and colleagues that PAX5/BSAP expression correlated with CD20 expression in myeloma, as PAX5/ BASP expression was only detectable in CD20positive cases. However, PAX5/BSAP and CD20 expression was not completely concordant. PAX5/ BSAP positivity was not detected in all CD20positive cases, and the staining intensity of PAX5/ BSAP was variable in most cases despite more uniform CD20 positivity demonstrated by flow cytometric immunophenotyping.

PAX5 mRNA levels analyzed by microarray were consistently low in the myeloma cells and did not predict PAX5/BSAP expression analyzed by immunohistochemistry. There was no significant difference in PAX5 mRNA levels between PAX5/BSAPpositive cases vs negative cases. The discordance between the gene and protein expression suggests that detected PAX5/BSAP likely represents a product of an earlier stage of differentiation that persisted after PAX5 gene expression has been repressed. The persistence of BSAP did not appear to inhibit the development of plasma cell phenotype and immunoglobulin secretion when PAX5 mRNA expression was repressed and PRDM1 was upregulated. This finding is in keeping with the observation that enforced expression of PRDM1 is sufficient to drive differentiation of $B$ cells to immunoglobulin-secreting plasma cells. ${ }^{17}$

The strong inverse correlation between PAX5 and PRDM1 mRNA levels in the myeloma cells, both CD20 positive and negative, recapitulates the gene expression patterns in normal plasma cells. ${ }^{18}$ However, since normal plasma cells are usually CD20

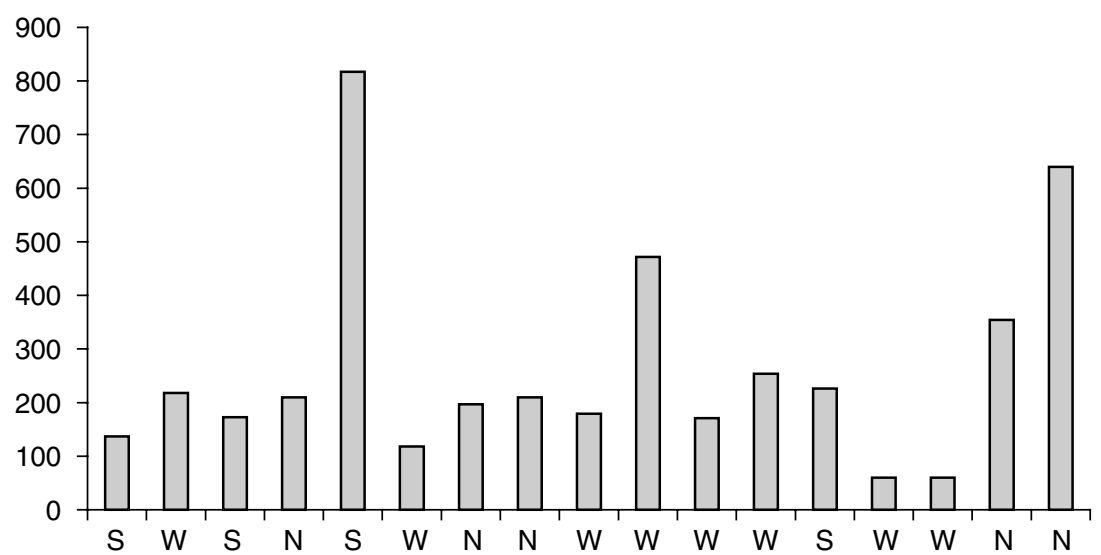

Figure 4 PAX5 mRNA levels (y-axis) assessed by microarray and corresponding BSAP intensity ( $x$-axis) assessed by immunohistochemistry in 17 cases of CD20-positive myeloma. S: Strong; W: Weak; N: Negative. 
negative, the asynchronous overexpression of PRDM1 and the B-cell associated marker CD20 in myeloma cells indicates disordered plasmacytic differentiation of B cells. Studies have shown that B cells from myeloma patients express low levels of full-length PAX5 but multiple isoforms that varied among individual patients. ${ }^{19} \mathrm{~A}$ splicing variant of PRDM1 mRNA is expressed at higher levels relative to the full-length form in the myeloma cells lines compared to the normal plasma cells. ${ }^{20}$ Thus, defective PAX5 expression and premature PRDM1 expression have been postulated to cause some proliferating $\mathrm{B}$ cells to prematurely differentiate to plasma cells in myeloma. The existence of CD20positive myelomas appears to support the hypothesis.

In conclusion, our studies show that PAX5/BSAP expression detected by immunohistochemistry is not restricted to B-cell lymphomas and leukemias. CD20-positive myelomas show variable expression of PAX5/BSAP, and those with strong and diffuse reactivity can present as a diagnostic pitfall mimicking B-cell neoplasms. PAX5 mRNA levels did not predict PAX5/BSAP expression but correlated inversely with PRDM1 mRNA levels in myeloma cells as in normal plasma cells. CD20-positive myeloma may be a useful model for studying disregulation of $P R D M 1$ and $P A X 5$ in pathogenesis of myeloma.

\section{References}

1 Hagman J, Wheat W, Fitzsimmons D, et al. Pax-5/ BSAP: regulator of specific gene expression and differentiation in B lymphocytes. Curr Top Microbiol Immunol 2000;245:169-194.

2 Lin KI, Angelin-Duclos C, Kuo TC, et al. Blimp-1dependent repression of Pax-5 is required for differentiation of $\mathrm{B}$ cells to immunoglobulin M-secreting plasma cells. Mol Cell Biol 2002;22:4771-4780.

3 Maier H, Hagman J. Roles of EBF and Pax-5 in B lineage commitment and development. Semin Immunol 2002;14:415-422.

4 Shapiro-Shelef M, Lin KI, McHeyzer-Williams LJ, et al. Blimp-1 is required for the formation of immunoglobulin secreting plasma cells and pre-plasma memory B cells. Immunity 2003;19:607-620.

5 Torlakovic E, Torlakovic G, Nguyen PL, et al. The value of anti-pax-5 immunostaining in routinely fixed and paraffin-embedded sections: a novel pan pre-B and B-cell marker. Am J Surg Pathol 2002;26:1343-1350.

6 Almeida J, Orfao A, Ocqueteau M, et al. High-sensitive immunophenotyping and DNA ploidy studies for the investigation of minimal residual disease in multiple myeloma. Br J Haematol 1999;107:121-131.

7 Grogan TM, Van Camp B, Kyle RA, et al. Plasma cell neoplasms. In: Jaffe ES, Harris NL, Stein H (eds). World Health Organization Classification of Tumors: Pathology and Genetics of Tumors of Hematopoietic and Lymphoid Tissues. IARC Press: Lyon, 2001.

8 Bartl R, Frisch B, Burkhardt R, et al. Bone marrow histology in myeloma: its importance in diagnosis, prognosis, classification and staging. Br J Haematol 1982;51:361-375.

9 Tricot G, Sawyer JR, Jagannath S, et al. Unique role of cytogenetics in the prognosis of patients with myeloma receiving high-dose therapy and autotransplants. J Clin Oncol 1997;15:2659-2666.

10 Lin P, Owens R, Tricot G, et al. Flow cytometric immunophenotypic analysis of 306 cases of multiple myeloma. Am J Clin Pathol 2004;121:482-488.

11 Zent CS, Zhan F, Schichman SA, et al. The distinct gene expression profiles of chronic lymphocytic leukemia and multiple myeloma suggest different anti-apoptotic mechanisms but predict only some differences in phenotype. Leuk Res 2003;27:765-774.

12 Zwollo P, Arrieta H, Ede K, et al. The Pax-5 gene is alternatively spliced during B-cell development. J Biol Chem 1997;272:10160.

13 Kehrl JH, Riva A, Wilson GL, et al. Molecular mechanisms regulating CD19, CD20 and CD22 gene expression. Immunol Today 1994;15:432-436.

14 Angelin-Duclos C, Cattoretti G, Lin KI, et al. Commitment of B lymphocytes to a plasma cell fate is associated with Blimp-1 expression in vivo. J Immunol 2000;165:5462-5471.

15 Robillard N, Avet-Loiseau H, Garand R, et al. CD20 is associated with a small mature plasma cell morphology and $t(11 ; 14)$ in multiple myeloma. Blood 2003;102:1070-1071.

16 Garcia-Sanz R, Orfao A, Gonzalez M, et al. Primary plasma cell leukemia: clinical, immunophenotypic, DNA ploidy, and cytogenetic characteristics. Blood 1999;93:1032-1037.

17 Shaffer AL, Lin KI, Kuo TC, et al. Blimp-1 orchestrates plasma cell differentiation by extinguishing the mature B cell gene expression program. Immunity 2002;17: 51-62.

18 Nagy M, Chapuis B, Matthes T. Expression of transcription factors Pu.1, Spi-B, Blimp-1, BSAP and Oct-2 in normal human plasma cells and in multiple myeloma cells. Br J Haematol 2002;116:429-435.

19 Borson ND, Lacy MQ, Wettstein PJ. Altered mRNA expression of Pax5 and Blimp-1 in B cells in multiple myeloma. Blood 2002;100:4629-4639.

20 Gyory I, Fejer G, Ghosh N, et al. Identification of a functionally impaired positive regulatory domain I binding factor 1 transcription repressor in myeloma cell lines. J Immunol 2003;170:3125-3133. 